\title{
Brief Report: Examining the Link Between Autistic Traits and Compulsive Internet Use in a Non-Clinical Sample
}

\author{
Catrin Finkenauer • Monique M. H. Pollmann • \\ Sander Begeer $\cdot$ Peter Kerkhof
}

Published online: 15 February 2012

(c) The Author(s) 2012. This article is published with open access at Springerlink.com

\begin{abstract}
Individuals with autism spectrum disorders or autistic traits may profit from Internet and computer-mediated interactions, but there is concern about their Internet use becoming compulsive. This study investigated the link between autistic traits and Internet use in a 2-wave longitudinal study with a non-clinical community sample $(n=390)$. As compared to people with less autistic traits, people with more autistic traits did not report a higher frequency of Internet use, but they were more prone to compulsive Internet use. For women, more autistic traits
\end{abstract}

\section{Finkenauer}

Social Psychology, VU University Amsterdam, Amsterdam,

The Netherlands

C. Finkenauer $(\bowtie)$

Department of Clinical Child and Family Studies, VU University

Amsterdam, van der Boechorststraat 1, Amsterdam 1082, BT,

The Netherlands

e-mail: c.finkenauer@vu.nl

M. M. H. Pollmann

Social Psychology, Tilburg University, Tilburg, The Netherlands

Present Address:

M. M. H. Pollmann

The Department of Communication and Information Sciences, Tilburg University, Tilburg, The Netherlands

\section{S. Begeer}

Department of Developmental Psychology, VU University

Amsterdam and School of Psychology, Amsterdam,

The Netherlands

S. Begeer

The University of Sydney, Sydney, Australia

P. Kerkhof

Communication Science, VU University Amsterdam,

Amsterdam, The Netherlands predicted an increase in compulsive Internet use over time. These results suggest that, despite its appeal for people with autistic traits, the Internet carries the risk of compulsive use.

Keywords Autism - AQ - Compulsive internet use . Computer $\cdot$ Autistic traits

\section{Introduction}

Individuals with autistic traits have impaired social and communicative skills that can cause difficulties in daily social interactions. Autistic traits have been related to individual differences in personality and behavior (Hoekstra et al. 2011). Internet and computer mediated interactions offer advantages for individuals with autistic traits because they enable them to interact with others in a safe and structured environment with reduced contextual, auditory, and visual cues. Despite its potential advantages, there is growing concern about excessive Internet use and its potential addictive properties, resulting in compulsive Internet use (van den Eijnden et al. 2008; Caplan 2003). Given their strong attraction to electronic screen media, it seems likely that people with autistic traits are especially prone to compulsive Internet use. Compulsive use of electronic screen media may harm offline social relationships rather than improve them (Kerkhof et al. 2011). This study seeks to investigate the link between autistic traits in a non-clinical sample and (compulsive) Internet use.

Most evidence for the beneficial effects of computeraided intervention stems from research on individuals with a clinical diagnosis of autism spectrum disorders (ASD). For children with ASD, positive effects have been reported on problem solving, vocabulary acquisition, and emotion 
recognition (Mineo et al. 2009; Ramdoss et al. 2011). Adults with ASD report the additional time to think of a response and the reduced pressure for eye-contact as important advantages of computer-mediated communications (Burke et al. 2010). In general, rule-based computermediated social interactions present a safe environment to practice and improve social functioning (Wilkinson et al. 2008). However, there may also be a downside to computer interactions, which has not received much attention yet. Because of its great appeal, media consumption may become excessive. For example, some children with ASD become obsessive about watching videos, causing serious difficulties for the rest of the family (Nally et al. 2000). In the current study, we will focus on autistic traits among individuals in the wider, a non-clinical population.

With the increased availability of the Internet nowadays, an increasing number of people spend considerable time online. In $2010,78.2 \%$ of the US population and $88.3 \%$ of the Dutch population were Internet users (www. Internetworldstats.com). Recent studies have shown that Internet usage can show addictive characteristics (Chou et al. 2005). These include preoccupation with the Internet, withdrawal reactions when Internet use is impossible, the failure to manage time spent online, and conflicts over Internet use with others (Kerkhof et al. 2011; Meerkerk et al. 2009).

Several studies found that the use of the Internet for communicational purposes in particular is related to compulsive Internet use (van den Eijnden et al. 2008). Students who prefer online communication over face-to-face communication are especially prone to problematic Internet use (Caplan 2003). People with autistic traits are likely to prefer Internet mediated communication over face-to-face communication because of the reduced complexity of the social situation (Burke et al. 2010). Thus, people with more autistic traits may show a higher risk of developing compulsive Internet use than people with less autistic traits.

Compulsive Internet use (CIU) can be defined as the inability to control online activity. This inability is reflected by continued use of the Internet despite the intention to stop, Internet use dominating a person's cognitions and behaviors, unpleasant emotions when Internet use is impossible, the use of the Internet to escape from negative feelings, and Internet use which results in conflict with others or with oneself (Meerkerk et al. 2009). CIU has a negative impact on psychological well-being and social functioning among adults and adolescents. Specifically, compulsive Internet use is related to feelings of loneliness (Morahan-Martin and Schumacher 2000). Also, it predicts depressive feelings (van den Eijnden et al. 2008) and declines in relationship quality (Kerkhof et al. 2011). Given these alarming effects of compulsive Internet use for social functioning and relationships, it is important to investigate whether there is a link between autistic traits and compulsive Internet use.

The present study investigates the link between autistic traits and Internet use in a longitudinal study among a nonclinical community sample. We examine both the frequency of Internet use and compulsive Internet use to show that people with autistic traits do not necessarily use the Internet more often, but that they are more likely to do so in a problematic way. We test these predictions in a large nonclinical community sample $(n=390)$ in order to examine the impact of autistic traits in the general population (for more information on the sample see Finkenauer et al. 2009; Kerkhof et al. 2011). Additionally, the longitudinal design of our study enables us to examine the relationship between autistic traits and Internet use over time. Although the results may not directly translate to people with clinical levels of ASD, they will provide a first indication whether Internet use can become problematic in that group.

\section{Method}

\section{Participants}

Participants were 195 married couples (190 at Time 2) in the Netherlands, who were studied twice with an interval of 1 year. At Time 1 husbands' mean age was 33.05 years $(\mathrm{SD}=4.86)$ and wives' mean age was 30.11 years $(\mathrm{SD}=4.25)$. Nearly all of the couples $(98.5 \%$ of the husbands and $96.4 \%$ of the wives) were Dutch. Both partners separately filled out questionnaires in the presence of a trained interviewer.

\section{Measures}

\section{Autism Spectrum Quotient (AQ)}

We used a Dutch version of the Abridged Autism-Spectrum Quotient (AQ-short) to measure autistic traits (Hoekstra et al. 2011; Pollmann et al. 2010). Internal consistency of the scale was good $(\alpha=.79)$.

\section{Frequency of Internet use}

To assess Frequency of Internet use, participants reported how many days per week they used the Internet for private purposes (as opposed to using the Internet for work). The answers were multiplied by scores on the question how many hours per day during these days they used the Internet for private purposes. This resulted in a score for the frequency of Internet use, ranging from 0 to 210 (h). Theoretically the measure has a maximum value of 168 ( 7 days in a week $* 24 \mathrm{~h}$ in a day). Consequently, the three 
participants who scored higher than the theoretical maximum (two at T1 and one at T2) probably misunderstood the question and were excluded from the analyses concerning frequency of Internet use.

\section{Compulsive Internet Use}

Compulsive Internet use was assessed using the Compulsive Internet Use Scale (Meerkerk et al. 2009). For reasons of space, we used a 5-item short version of the original 14-item scale. We averaged the factor loadings reported in the three studies that Meerkerk et al. (2009) reported in their scale construction. Across the three studies, the authors found a one factor solution for compulsive Internet use. We selected the five items with the highest average loadings on the one factor. The items were slightly modified because the original items were aimed at adolescents rather than at an adult population. The items are: "How often.... (1) do you find it difficult to stop using the Internet when you are online? (2) do you continue to use the Internet despite your intention to stop?, (3) do you prefer to use the Internet instead of spending time with others (e.g., partner, children, parents, friends)? (4) are you short of sleep because of the Internet?, (5) do you feel restless, frustrated, or irritated when you cannot use the Internet?" ( $\alpha=.61$ for wives and $\alpha=.77$ for husbands).

\section{Results}

\section{Descriptive Analyses}

We first conducted descriptive analyses. Husbands had more autistic traits than wives, $t(189)=4.63, p<.001$. Over time, participants showed a decreased frequency of Internet use (13.21 vs. 9.54), $F(1,152)=9.83, p<.002$, $\eta^{2}=.061$. No main effect of sex or an interaction with sex emerged. There was a main effect of sex on Compulsive Internet use, $F(1,177)=21.10, p<.001, \eta^{2}=.11$; men $(M=1.75, \mathrm{SD}=0.63)$ reported more Compulsive Internet use than women, $(M=1.50, \mathrm{SD}=0.48)$. There was no main effect of time, but a significant interaction between sex and time, $F(1,177)=8.09, p=.005, \eta^{2}=.04$. Compulsive Internet use tended to decrease over time for men $(1.78$ vs. 1.72), $F(1,177)=3.66, \quad p=.057$, $\eta^{2}=.020$, but increased for women (1.47 vs. 1.54$), F(1$, 177) $=4.52, p=.04, \eta^{2}=.025$.

Cross-Sectional Relation Between Autistic Traits and Internet Use

To investigate whether autistic traits were associated with frequency of Internet use and compulsive Internet use cross-sectionally, we ran two mixed model analyses to predict both couple members' frequency of Internet use and compulsive Internet use with their score on the AQ, taking into account interdependence between partners. Frequency of Internet use was not associated with autistic traits $(\beta=-.05, p=.77)$ or $\operatorname{sex}(\beta=-.02, p=.88)$ or the interaction of autistic traits and $\operatorname{sex}(\beta=.06, p=.60)$. This indicates that there is no statistically significant association between frequency of internet use and level of autistic traits. Compulsive Internet use was related to autistic traits; people with more autistic traits reported more compulsive Internet use than people with less autistic traits $(\beta=.48, p=.002)$. There was no significant interaction between autistic traits and $\operatorname{sex}(\beta=-.15, p=.12)$. Compulsive Internet use was also related to sex; men reported more compulsive Internet use than women $(\beta=-.41, p<.001)$.

\section{Longitudinal Relation Between Autistic Traits} and Internet Use

To investigate whether autistic traits and sex predict change in Internet use over time, we conducted residualized lagged analyses. That is, we predicted frequency of Internet use and compulsive Internet use at Time 2 with AQ-scores at Time 1 and sex, controlling for Internet use and compulsive Internet use scores at Time 1 .

\section{Frequency of Internet Use}

Indicating that frequency of Internet is relatively stable over time, frequency of Internet use at Time 2 was predicted by frequency of Internet use at Time $1,(\beta=.38$, $p=.029)$. No other effects were significant.

\section{Compulsive Internet Use}

Compulsive Internet use also showed considerable stability over time, with compulsive Internet use at Time 1 predicting compulsive Internet use at Time $2(\beta=.68$, $p<.001)$. No other main effects emerged. We did find a significant interaction of compulsive Internet use at Time 1 with autistic traits $(\beta=-.17, p=.001)$ and a significant 3-way interaction between compulsive Internet use at Time 1 , autistic traits, and $\operatorname{sex}(\beta=.14, p=.045)$. We examined this interaction separately for each sex. For men, compulsive Internet use at Time 1 predicted an increase in compulsive Internet use at Time $2(\beta=.78, p<.001)$. For women, we found both a main effect of compulsive Internet use at Time $1(\beta=.68, p<.001)$ and a significant interaction of compulsive Internet use at Time 1 with autistic traits $(\beta=-.17, p=.001)$. Simple slopes analyses revealed that for women with relatively low levels of 
initial compulsive Internet use more autistic traits predicted an increase of compulsive Internet use at Time $2(\beta=.21$, $p<.01)$. For women with relatively high levels of initial compulsive Internet use autistic traits did not predict later levels of compulsive Internet use $(\beta=.06, p=.57)$.

\section{Discussion}

The findings of the current investigation provide the first empirical evidence that autistic traits are related to compulsive Internet use in men and women. That is, at a given time, people with more autistic traits report more compulsive Internet use. Somewhat surprisingly, autistic traits only predicted an increase of compulsive Internet use in women with low earlier compulsive Internet use. Why do we find this sex difference? In our study, men showed higher overall compulsive Internet use than women and the increase in compulsive Internet use occurred only for women with more autistic traits and low compulsive Internet use to begin with. It is possible that once people surpass a certain level of compulsive Internet use, autistic traits may not add to increases in compulsive Internet use. In these cases, only earlier compulsive behavior predicts increases in later compulsive behavior. When compulsive Internet use is low (as was the case for certain women in our sample), however, autistic traits may facilitate the development of compulsive Internet use. Given the correlational design of our study, the exact causal relationship between autistic traits and CIU remains unknown.

In line with earlier research in populations with a clinical diagnosis of ASD (Mineo et al. 2009), we show that certain media can become too attractive. Although the Internet facilitates certain forms of social contact in that it provides a safe and structured environment, there is a risk that people with autistic traits will use the Internet compulsively, which may be harmful to their 'offline' social relationships. Several findings provide indirect support for this suggestion. Because people with autistic traits are inhibited and less competent in face-to-face interactions, they may prefer the more protected interactions via Internet, similar to socially anxious people (Schouten et al. 2007). Despite their reluctance to real life interactions, autistic traits are not linked to a desire to be socially excluded. On the contrary, children with a clinical diagnosis of ASD often experience feelings of loneliness (Bauminger et al. 2003).

In addition, autistic traits are linked with a tendency for repetitive and stereotypical behavior, suggesting that higher levels of compulsive Internet use may be directly related to autistic traits. A preference of online over offline interactions, in turn, may harm existing relationships in the long-run (Kerkhof et al. 2011). This is especially troublesome given that friends and family are the main sources of everyday social support. Thus, although the Internet may be an appealing and helpful medium for people with autistic traits, it is important to closely monitor whether being online is becoming compulsive to make sure that Internet use does not affect offline social relationships in a negative manner.

On the positive side, the reduced contextual, auditory, and visual cues in online interactions (Trepagnier et al. 2006, 2011) diminish the constraints that individuals with autistic traits experience in offline interactions. Also, the Internet provides people with autistic traits with the opportunity to control the information they want to reveal about themselves. This increase in control over self-presentation and self-disclosure may be attractive to individuals with autistic traits who experience social hindrances in face-to-face interactions.

We do not claim that Internet use among people with autistic traits always leads to detrimental effects. Our goal here was to demonstrate, to our knowledge for the first time, that autistic traits can be associated with problematic internet use. It is unclear at this point whether the effect generalizes to clinical samples with ASD, and how compulsive Internet use may reduce the quality of their existing relationships as Kerkhof et al. (2011) suggest.

Another question that would be interesting to explore is whether the type of activity on the Internet matters. To illustrate, research shows that the quality of adolescents' relationships profits from online communications with existing friends, but not from the use of Internet for entertainment purposes or in chatrooms (Valkenburg and Peter 2009). More research is needed to examine whether the link between autistic traits and compulsive Internet use is moderated by the type of Internet use people engage in. Awaiting future research, we conclude that although Internet is an appealing and helpful medium for people with autistic traits, its use may also come at a prize. Especially among people with autistic traits it therefore is important to closely monitor their Internet use to make sure that it does not interfere 'offline' contacts.

Acknowledgments This research was supported by a grant to the first author from the Netherlands Organization for Scientific Research (No. 452-05-322).

Open Access This article is distributed under the terms of the Creative Commons Attribution License which permits any use, distribution, and reproduction in any medium, provided the original author(s) and the source are credited. 


\section{References}

Bauminger, N., Shulman, C., \& Agam, G. (2003). Peer interaction and loneliness in high-functioning children with autism. Journal of Autism Developmental Disorders, 33, 489-507.

Burke, M., Kraut, R., \& Williams, D. (2010). Social use of computermediated communication by adults on the autism spectrum. Paper presented at the ACM conference on Computer supported cooperative work, Savannah, Georgia, USA.

Caplan, S. E. (2003). Preference for online social interaction: A theory of problematic Internet use and psychosocial well-being. Communication Research, 30, 625-648. doi:10.1177/00936502 03257842.

Chou, C., Condron, L., \& Belland, J. C. (2005). A review of the research on internet addiction. Educational Psychology Review, 17(4), 363-388. doi:10.1007/s10648-005-8138-1.

Finkenauer, C., Kerkhof, P., Righetti, F., \& Branje, S. J. T. (2009). Living together apart: Perceived concealment as signal of exclusion in marital relationships. Personality and Social Psychological Bulletin, 35, 1410-1422.

Hoekstra, R. A., Vinkhuyzen, A. A. E., Wheelwright, S., Bartels, M., Boomsma, D. I., Baron-Cohen, S., et al. (2011). The construction and validation of an abridged version of the autism-spectrum quotient (AQ-Short). Journal of Autism and Developmental Disorders, 41, 589-596.

Kerkhof, P., Finkenauer, C., \& Muusses, L. D. (2011). Relational consequences of compulsive Internet use: A longitudinal study among newlyweds. Human Communication Research. doi: 10.1111/j.1468-2958.2010.01397.x/pdf.

Meerkerk, G. J., Van Den Eijnden, R. J. J. M., Vermulst, A. A., \& Garretsen, H. F. L. (2009). The Compulsive Internet Use Scale (compulsive Internet useS): Some psychometric properties. CyberPsychology \& Behavior, 12, 1-6. doi:10.1089/cpb.2008. 0181.

Mineo, B. A., Ziegler, W., Gill, S., \& Salkin, D. (2009). Engagement with electronic screen media among students with Autism Spectrum Disorders. Journal of Autism and Developmental Disorders, 39, 172-187.

Morahan-Martin, J., \& Schumacher, P. (2000). Incidence and correlates of pathological Internet use among college students.
Computers in Human Behavior, 16, 13-29. doi:10.1016/s07475632(99)00049-7.

Nally, B., Houlton, B., \& Ralph, S. (2000). Researches in brief: The management of television and video by parents of children with autism. Autism, 4, 331-337. doi:10.1177/1362361300004003008.

Pollmann, M. M. H., Finkenauer, C., \& Begeer, S. (2010). Mediators of the link between autistic traits and relationship satisfaction in a non-clinical sample. Journal of Autism and Developmental Disorders, 40(4), 470-478. doi:10.1007/s10803-009-0888-z.

Ramdoss, S., Mulloy, A., Lang, R., O’Reilly, M., Sigafoos, J., Lancioni, G., et al. (2011). Use of computer-based interventions to improve literacy skills in students with autism spectrum disorders: A systematic review. Research in Autism Spectrum Disorders, 5, 1306-1318.

Schouten, A. P., Valkenburg, P. M., \& Peter, J. (2007). Precursors and underlying processes of adolescents' online self-disclosure: Developing and testing an "Internet-attribute-perception" model. Media Psychology, 10, 292-314.

Trepagnier, C. Y., Olsen, D. E., Boteler, L., \& Bell, C. A. (2011). Virtual conversation partner for adults with autism. Cyberpsychology Behavior and Social Setworking, 14, 21-27.

Trepagnier, C. Y., Sebrechts, M. M., Finkelmeyer, A., Stewart, W., \& Woodford, J. (2006). Acceptance of a virtual social environment by pre-schoolers with autism spectrum disorder. Cyberpsychology \& Behavior, 9, 723.

Valkenburg, P. M., \& Peter, J. (2009). Social consequences of the Internet for adolescents: A decade of research. Current Directions in Psychological Science, 18, 1-5. doi:10.1111/j.14678721.2009.01595.x.

Van den Eijnden, R. J. J. M., Meerkerk, G.-J., Vermulst, A. A., Spijkerman, R., \& Engels, R. C. M. E. (2008). Online communication, compulsive Internet use and psychosocial well-being among adolescents: A longitudinal study. Developmental Psychology, 44, 655-665. doi:10.1037/0012-1649.44.3. 655.

Wilkinson, N., Ang, R. P., \& Goh, D. H. (2008). Online video game therapy for mental health concerns: A review. International Journal of Social Psychiatry, 54(4), 370-382. doi:10.1177/0020 764008091659. 